\title{
Canonical formalism of dissipative fields in thermo field dynamics
}

T. Arimitsu

Institute of Physics, University of Tsukuba, Ibaraki 305, Japan

$\mathrm{H}$. Umezawa and Y. Yamanaka

Theoretical Physics Institute, Department of Physics, University of Alberta, Edmonton, Alberta T6G 2J1, Canado

(Received 30 December 1986; accepted for publication 1 July 1987)

For the stationary case the canonical formalism of thermally dissipative fields with both positive- and negative-frequency parts is constructed. This formulation enables one to follow the self-consistent renormalization scheme which creates the dissipation spontaneously. The self-interacting $\varphi^{3}$ model is examined as an example of the spontaneous creation of dissipation. The parameter $\alpha$ appearing in the thermal state conditions as well as observables independent of the choice of $\alpha$ are discussed.

\section{INTRODUCTION}

The quantum field theory provides us with a useful formalism for quantum systems with infinite degrees of freedom. A program of reformulating the nonequilibrium quantum statistical mechanics with infinite degrees of freedom in the terminology of quantum field theory has been developed by extending thermo field dynamics (TFD) which is quantum field theory with thermal degrees of freedom. ${ }^{1-8}$ This extended TFD was shown to be equivalent to the density matrix formalism with the Liouville equation. ${ }^{1}$ The extended TFD has so far been formulated in terms of harmonic oscillators. The purpose of this paper is to reformulate the extended TFD as a formalism for quantum field theory.

As is now widely known, TFD is built on the concepts of thermal doublets, the thermal vacuum, and the Hamiltonian. ${ }^{9,10}$ Thus with any operator $A$ there is associated another operator $\widetilde{A}$ which is called the tilde conjugate of $A$. The doublet $A^{\mu}$ with $A^{1}=A$ and $A^{2}=\widetilde{A}^{\dagger}$ is called the thermal doublet. The tilde conjugation rules are summarized as

$$
\begin{aligned}
& {[A B]^{\sim}=\widetilde{A B}^{\sim},} \\
& {\left[c_{1} A+c_{2} B\right]^{\sim}=c_{1}^{*} \tilde{A}+c_{2}^{*} \widetilde{B},} \\
& {\left[A^{\dagger}\right]^{\sim}=\widetilde{A}^{\dagger},} \\
& {[\widetilde{A}]^{\sim}=\sigma A,} \\
& |0\rangle^{\sim}=|0\rangle, \\
& \left\langle\left. 0\right|^{\sim}=\langle 0|,\right.
\end{aligned}
$$

where $|0\rangle$ and $\langle 0|$ are the thermal vacua, $\sigma=+1(-1)$ for bosonic (fermionic) $A$ and the $c_{i}$ 's are $c$ numbers.

The Hamiltonian is constructed as follows. When a system of quantum fields is given, we write the Lagrangian density $\mathscr{L}[\psi]$ from which the canonical Hamiltonian $H[\psi]$ follows through the well-known route. Then we construct $\widetilde{H}$ by applying the tilde conjugation rules to $H$. The Hamiltonian is then given by

$$
\hat{H}=H-\widetilde{H} \text {. }
$$

This statement is quite general. It covers all thermal situations, both equilibrium and nonequilibrium.

To make our consideration explicit, let us consider a nonequilibrium transition from a situation of normal con- ductivity to one of superconductivity. The initial situation is described by the normal quasielectron field, while the final situation is described by the superconducting quasielectron field. The intermediate situation is described by a time-dependent quasiparticle field (or a renormalized field). Thus a reasonable computational method may be the perturbative calculation in which the unperturbed representation corresponds to the quasiparticle field. Since the key mechanism in nonequilibrium phenomena is thermal dissipation, the quasiparticle field under consideration should be dissipative. In the sense that the field equations of the quasiparticles are linear and homogeneous differential equations, they can be said to be free. However, since they are dissipative, they are not really free. We have therefore called them semifree. Using this terminology we use semifree fields for the unperturbed representation.

It may be important to note that the dissipative effect is important even in a stationary case. Consider a situation in which the ground state is an equilibrium state. Even in such a case the excited states approach the ground state dissipatively. Then although the thermal averages of observables are independent of time, the two-point functions such as the Green's functions or correlation functions exhibit dissipative effects caused by the contributions due to the excited states. This means that the Hamiltonian for the semifree fields should contain thermally dissipative terms.

It is obvious from the above consideration that the perturbative calculation formalism requires knowledge of the general structure of the semifree field theory as its beginning. Once we know the Hamiltonian say $\left(\hat{H}^{0}\right)$ of the semifree field, then crudely speaking $\widehat{H}-\widehat{H}^{0}$ acts as the interaction Hamiltonian. Then the Feynman diagram method tells us how to proceed in the perturbative computation. Just as the physical mass is determined by the self-consistent renormalization method that leads to mass equations, the dissipative constant is to be determined by the self-consistent renormalization method that leads to equations for dissipative coefficients. When the latter equations give rise to a nonvanishing dissipative coefficient the phenomenon is called the spontaneous creation of dissipation..$^{5-7}$ In the framework of TFD this phenomenon is expected to happen in almost all cases. 
In a simple example it was shown by an exact solution. ${ }^{7}$

Since the Hamiltonian of a semifree system consists of the thermal doublets, the dissipative term has the form of a $2 \times 2$ thermal matrix like $\bar{a}^{\mu} A^{\mu \nu} a^{\nu}$. At first glance it may seem that the structure of the matrix $A$ is quite arbitrary, but in fact the matrix $A$ was found to have a very particular form. In particular, the existence of the off-diagonal elements of $A$, which combine tilde and nontilde operators, indicates that this dissipation is a thermal effect.

So far the study of the structure of semifree systems has been made only for harmonic oscillator-type operators. One purpose of this paper is to formulate the theory in terms of semifree fields instead of in terms of harmonic oscillatortype operators.

It is worth commenting on the dynamical map in TFD (i.e., the expression of Heisenberg fields in terms of certain free fields). The thermal instability giving rise to the imaginary terms in the self-energy forces us to abandon the dynamical map in terms of the usual asymptotic free fields based on the stable particle picture. In fact a no go theorem ${ }^{11}$ states that the $S$ matrix is trivial when the dynamical maps are expressed in terms of asymptotic free fields in TFD. Instead we express the dynamical map in TFD in terms of the semifree fields:

$$
\psi \stackrel{\mathrm{w}}{=} \psi[\varphi],
$$

where $\psi$ and $\varphi$ are the Heisenberg field and the semifree field, respectively. ${ }^{8}$ The symbol $\stackrel{\mathrm{w}}{=}$ is the weak equality (i.e., the equality of matrix elements in reference to the Fock space of the semifree field $\varphi$ ). We feel that the reasons why (1.3) is possible are the negative energy of the tilde quantum and the infinite number of degrees of freedom. The creation or annihilation of a tilde quantum describes a change in the thermally excited background field and is therefore not observed as particle creation or annihilation. Intuitively speaking, the nontilde particles are acting under the influence of the thermally excited background field. This is the reason for the thermal instability of particles; they become unstable through communication with the thermal background field. The eigenvalues of $\hat{H}^{0}$ with imaginary dissipative part may have nothing to do with eigenvalues of $\hat{H}$ and furthermore $\hat{H}$ itself has no eigenstate in the present realization space (i.e., the Fock space of $\varphi$ ). The latter statement reminds us of the fact that a generator $G$ has no eigenstates in the representation space in which the symmetry generated by $G$ is spontaneously broken in quantum field theory.

It has been shown that there is a parameter $\alpha$ in TFD with the property that the physical results are independent of $\alpha$. The Keldysh-Schwinger formalism ${ }^{12}$ corresponds to the choice $\alpha=1$, while the so-called equilibrium TFD uses $\alpha=\frac{1}{2},{ }^{9,10}$ In modern TFD for operators of harmonic oscillators the choice of $\alpha$ has been left undetermined. In Sec. III the structure of $\alpha$ transformations will be presented.

In Sec. IV the structure of the semifree fields will be presented. Here we restrict our consideration to stationary situations only, and we choose $\alpha=\frac{1}{2}$. The semifree field is expressed in terms of an orthonormalized complete set of wave functions which satisfy the canonical sum rule; ${ }^{8}$ this situation is exactly the same as the one for the usual free field theory. In this way the semifree fields acquire the usual $c a-$ nonical formalism. The semifree field theory with arbitrary choice of $\alpha$ in both stationary and time-dependent situations will be presented in a separate paper.

In Sec. $\mathrm{V}$ we analyze a self-interacting scalar field in a stationary situation. It will be explicitly shown how the selfconsistent equation for the dissipative coefficient emerges from the renormalization calculation. Althought this renormalization method has been presented only in consideration of a very simple model, the general method for choosing the renormalization point in stationary situations will be illustrated.

As a preparation for the analysis in this paper, in Sec. II, there will be given a very brief summary of the semifree oscillator theory. ${ }^{5}$

\section{TFD IN TERMS OF SEMIFREE OSCILLATOR}

We consider the oscillator variables classified by the "momentum" $\mathbf{k}$, i.e., $a(\mathbf{k})$ and $\tilde{a}(\mathbf{k})$. The thermal doublets are

$$
\begin{array}{ll}
a^{\mu}(\mathbf{k}): & a^{1}(\mathbf{k})=a(\mathbf{k}), \quad a^{2}=\tilde{a}^{\dagger}(\mathbf{k}), \\
\bar{a}^{\mu}(\mathbf{k}): & \bar{a}^{1}(\mathbf{k})=a^{\dagger}(\mathbf{k}), \quad \bar{a}^{2}=-\sigma \tilde{a}(\mathbf{k}) .
\end{array}
$$

The thermal vacuum is denoted by $|0\rangle$ and $\langle 0|$. We can write

$$
\bar{a}^{\mu}(\mathbf{k})=a^{\dagger}(\mathbf{k}) \tau_{\sigma}
$$

with

$$
\tau_{\sigma}=\left(\begin{array}{cc}
1 & 0 \\
0 & -\sigma
\end{array}\right)
$$

We have

$$
\left[a^{\mu}(\mathbf{k}), \bar{a}^{\nu}(\mathbf{1})\right]_{\sigma}=\delta_{\mu \nu} \delta(\mathbf{k}-\mathbf{l})
$$

with $\sigma= \pm 1$.

In this section and Sec. III, we include both the stationary and time-dependent situations in our considerations. The time evolution of operator is given by

$$
\begin{aligned}
& a(t, \mathbf{k})^{\mu}=\widehat{S}^{-1}(t) a(\mathbf{k})^{\mu} \hat{S}(t), \\
& \bar{a}(t, \mathbf{k})^{\mu}=\widehat{S}^{-1}(t) \bar{a}(\mathbf{k})^{\mu} \widehat{S}(t),
\end{aligned}
$$

with $\widehat{S}(t=0)=1$ and

$$
\partial_{t} \hat{S}(t)=-i \hat{H}{ }_{t}^{0} \hat{S}(t) \text {. }
$$

The relations in (2.4) are consistent with the tilde conjugation rules (1.1) when and only when the semifree Hamiltonian $\hat{H}_{t}^{\circ}$ is tildian,

$$
\left(i \hat{H}_{t}^{0}\right)^{\sim}=i \hat{H}_{t}^{0} \text {. }
$$

In the quantum field theory without thermal freedom the vacuum is empty of physical particles, implying $a(\mathbf{k})|0\rangle=0$. When we have the thermal degree of freedom, the vacuum contains thermally excited particles. Thus $a(\mathbf{k})|0\rangle \neq 0$. It has been shown ${ }^{1}$ that the vacuum is conditioned by the thermal state conditions which read as

$$
\begin{aligned}
& a(t, \mathbf{k})^{1}|0\rangle=f^{\alpha}(t, \mathbf{k}) a(t, \mathbf{k})^{2}|0\rangle, \\
& \langle 0| \bar{a}(t, \mathbf{k})^{1}=-\sigma f^{1-\alpha}(t, \mathbf{k})\langle 0| \bar{a}(t, \mathbf{k})^{2},
\end{aligned}
$$

with a real and positive function $f(t, \mathbf{k})$ and $0 \leqslant \alpha \leqslant 1$. The parameter $\alpha$ may depend on time. 
Considering the tilde conjugation rules (1.1) we have from (2.7)

$$
\begin{aligned}
\bar{a}(t, \mathbf{k})^{2}|0\rangle & =-f^{\alpha}(t, \mathbf{k}) \bar{a}(t, \mathbf{k})^{1}|0\rangle, \\
\langle 0| a(t, \mathbf{k})^{2} & =\sigma f^{1-\alpha}(t, \mathbf{k})\langle 0| a(t, \mathbf{k})^{1} .
\end{aligned}
$$

The relations (2.7) and (2.8) give the complete set of $[\alpha]$ representation thermal state conditions for the semifree oscillators. In deriving (2.8) we have used the assumption that $f(t, \mathbf{k})$ is real which is equivalent to considering only systems with real particle number

$$
\begin{aligned}
n(t, \mathbf{k}) & =\left\langle 0\left|\bar{a}(t, \mathbf{k})^{1} a(t, \mathbf{k})^{1}\right| 0\right\rangle \\
& =f(t, \mathbf{k}) /[1-\sigma f(t, \mathbf{k})],
\end{aligned}
$$

where (2.7) and (2.8) have been used. When $f(t, \mathbf{k})$ is independent of $t$ [i.e., $f(t, \mathbf{k})=f(\mathbf{k})$ ], we state that the situation is stationary.

Since $\widehat{S}(t)$ is not necessarily unitary, we used the symbol $\dagger \dagger$ instead of $\dagger$. Note that the nonequilibrium TFD presented in Refs. 1 and 2 used the $[\alpha=1]$ representation, while the equilibrium TFD in Refs. 9 and 10 employed the $\left[\alpha=\frac{1}{2}\right]$ representation. The $[\alpha=0]$ representation corresponds to the mirror space. The physical quantities, the detailed definition of which will be given in the next section, are independent of the choice of $\alpha$.

It follows from the thermal state conditions that $\hat{H}_{t}^{o}$ has the following structure:

$$
\begin{aligned}
\hat{H}_{t}^{0}= & \int d^{3} k \vec{a}(\mathbf{k})^{\mu}\left[\omega(t, \mathbf{k}) \delta^{\mu v}-i \kappa(t, \mathbf{k}) A(t, \mathbf{k})^{\mu v}\right. \\
& +\sigma\left\{\partial_{t} n(t, \mathbf{k})\right\} \tau(t, \mathbf{k})^{\mu v} \\
& \left.+\left\{\partial_{t} \ln f(t, \mathbf{k})^{(1-\alpha) / 2}\right\} \tau_{3}^{\mu \nu}\right] a(\mathbf{k})^{v} .
\end{aligned}
$$

Here $\kappa(t, \mathbf{k})$ is the dissipative coefficient. The matrices $A$ and $\tau$ are

$$
A(t, \mathbf{k})=\left[\begin{array}{cc}
1+2 \sigma n(t, \mathbf{k}) & -2 f^{\alpha-1}(t, \mathbf{k}) n(t, \mathbf{k}) \\
2 \sigma f^{1-\alpha}(t, \mathbf{k})[1+\sigma n(t, \mathbf{k})] & -[1+2 \sigma n(t, \mathbf{k})]
\end{array}\right],
$$

$$
\tau(t, \mathbf{k})=\left[\begin{array}{cc}
1 & -\sigma f^{\alpha-1}(t, \mathbf{k}) \\
\sigma f^{1-\alpha}(t, \mathbf{k}) & -1
\end{array}\right]
$$

and the $\tau_{i}$ 's $(i=1,2,3)$ are Pauli matrices.

The thermal state conditions (2.7) and (2.8) have the form

$$
\begin{aligned}
& \gamma(t, \mathbf{k})^{1}|0\rangle=\bar{\gamma}(t, \mathbf{k})^{2}|0\rangle=0, \\
& \langle 0| \gamma(t, \mathbf{k})^{2}=\langle 0| \bar{\gamma}(t, \mathbf{k})^{1}=0,
\end{aligned}
$$

where

$$
\begin{aligned}
& \gamma(t, \mathbf{k})^{\mu}=B(t, \mathbf{k})^{\mu \nu} a(t, \mathbf{k})^{v}, \\
& \bar{\gamma}(t, \mathbf{k})^{\mu}=\bar{a}(t, \mathbf{k})^{v} B^{-1}(t, \mathbf{k})^{v \mu},
\end{aligned}
$$

with

$$
\begin{aligned}
B(t, \mathbf{k})= & {[1-\sigma f(t, \mathbf{k})]^{-1 / 2} } \\
& \times\left[\begin{array}{cc}
1 & -f^{\alpha}(t, \mathbf{k}) \\
-\sigma f^{1-\alpha}(t, \mathbf{k}) & 1
\end{array}\right] .
\end{aligned}
$$

In defining $\gamma^{\mu}$ and $\bar{\gamma}^{\mu}$, we have imposed the condition that $\operatorname{det} B=1$. Equations (2.14) lead to

$$
\left[\gamma^{\mu}(t, \mathbf{k}), \bar{\gamma}^{\nu}(t, \mathbf{l})\right]_{\sigma}=\delta_{\mu \nu} \delta(\mathbf{k}-\mathbf{l}) .
$$

The equations in (2.13) indicate that $\gamma(t, \mathbf{k})^{1}$ and $\bar{\gamma}(t, \mathbf{k})^{2}$ are the annihilation operators while $\gamma(t, \mathbf{k})^{2}$ and $\bar{\gamma}(t, \mathbf{k})^{1}$ are the creation operators. We can prove

$$
B^{-1}(t, \mathbf{k})=\tau_{3} B(t, \mathbf{k}) \tau_{3} .
$$

It has been shown that $\gamma(t, \mathbf{k})^{\mu}$ and $\bar{\gamma}(t, \mathbf{k})^{\mu}$ are the eigenfunctions of the form

$$
\begin{aligned}
\gamma(t, \mathbf{k})^{\mu}= & {\left[W(t, 0, \mathbf{k}) \exp \int_{0}^{t} d \tau\{-i \omega(\tau, \mathbf{k})\right.} \\
& \left.\left.-\tau_{3} \kappa(\tau, \mathbf{k})\right\}\right]^{\mu v} \gamma(t=0, \mathbf{k})^{\nu}
\end{aligned}
$$


The calculation of the unperturbed causal two-point function,

$$
G(t, s, \mathbf{k})^{\mu \nu} \delta(\mathbf{k}-\mathbf{l})=-i\left\langle 0\left|T\left[a(t, \mathbf{k})^{\mu} \bar{a}(s, \mathbf{l})^{\nu}\right]\right| 0\right\rangle,
$$

can be made by means of the method of the Wick-type formula. The result has been obtained. It is

$$
G(t, s, \mathbf{k})^{\mu v}=\left[B(t, \mathbf{k}) \mathscr{G}(t, s, \mathbf{k}) B^{-1}(s, \mathbf{k})\right]^{\mu v},
$$

where

$$
\mathscr{G}(t, s, \mathbf{k})^{\mu \nu} \delta(\mathbf{k}-l)=-i\left\langle 0\left|T\left[\gamma(t, \mathbf{k})^{\mu} \bar{\gamma}(s, l)^{\nu}\right]\right| 0\right\rangle,
$$

whose elements are explicitly given by

$$
\begin{aligned}
& \mathscr{G}(t, s, \mathbf{k})^{11}=z(t, s, \mathbf{k}) G^{r}(t, s, \mathbf{k}), \\
& \mathscr{G}(t, s, \mathbf{k})^{22}=z(s, t, \mathbf{k}) G^{\alpha}(t, s, \mathbf{k}),
\end{aligned}
$$

and $\mathscr{G}(t, s, \mathbf{k})^{12}=\mathscr{G}(t, s, \mathbf{k})^{21}=0$, with

$$
\begin{aligned}
G^{r}(t, s, \mathbf{k})= & -i \theta(t-s) \exp \left[\int_{s}^{t} d \tau\right. \\
& \times\{-i \omega(\tau, \mathbf{k})-\kappa(\tau, \mathbf{k})\}], \\
G^{a}(t, s, \mathbf{k})= & i \theta(s-t) \exp \left[\int_{s}^{t} d \tau\right. \\
& \times\{-i \omega(\tau, \mathbf{k})+\kappa(\tau, \mathbf{k})\}] .
\end{aligned}
$$

\section{THE $\alpha$ TRANSFORMATION AND OBSERVABLES}

As is seen from the arguments in the previous section, we have infinite ways of representing a thermal situation through the thermal state conditions. This freedom was indicated by $\alpha$ in the thermal state conditions (2.7) and (2.8). (In the density operator formalism ${ }^{13}$ the $\alpha$ freedom arises from the trace formula $\operatorname{Tr}[\rho A]=\operatorname{Tr}\left[\rho^{1-\alpha} A \rho^{\alpha}\right]$ with any operator $A$ and the Liouville equation of the form $\dot{\rho}^{\alpha}=-i\left[\rho^{\alpha}, H\right]$.) According to (2.4), (2.5), and (2.10), the parameters and operators appearing in the free dissipative Hamiltonian also depend on $\alpha$, and therefore $\widehat{H}^{\circ}(t)$,

$$
\hat{H}^{0}(t)=\widehat{S}^{-1}(t) \hat{H}_{t}^{0} \widehat{S}(t)
$$

should be denoted by $\widehat{H}_{\alpha}^{\circ}(t)$ :

$$
\widehat{H}_{\alpha}^{o}(t)=\widehat{H}^{o}\left(a_{\alpha}(t), \bar{a}_{\alpha}(t) ; \alpha\right) .
$$

Thus both the thermal state conditions and the free dissipative Hamiltonian depend on $\alpha$. The fact that the thermal state conditions depend on $\alpha$ implies that the thermal vacuum also depends on $\alpha$. Thus we should write $\left|0_{\alpha}\right\rangle$ and $\left\langle 0_{\alpha}\right|$. However, in the following sections, the suffix $\alpha$ in the Hamiltonian and the thermal vacua will be mostly omitted.

The observable results, however, should be independent of $\alpha$. Therefore it is convenient to find the transformation which changes $\alpha$ as $\alpha \rightarrow \alpha^{\prime}$ in order to identify the observable operators.

Let $T(0)$ denote the operator inducing the following transformation:

$$
\left|0_{\alpha^{\prime}}\right\rangle=T^{-1}(0)\left|0_{\alpha}\right\rangle, \quad\left\langle 0_{\alpha^{\prime}}\right|=\left\langle 0_{\alpha}\right| T(0) .
$$

Without loss of generality we can prepare all of the operators in such a way that they become independent of $\alpha$ at $t=0$ :

$$
\begin{aligned}
& a(0)^{\mu} \equiv a_{\alpha}(0)^{\mu}=a_{\alpha^{\prime}}(0)^{\mu}, \\
& \bar{a}(0)^{\mu} \equiv \bar{a}_{\alpha}(0)^{\mu}=\bar{a}_{\alpha^{\prime}}(0)^{\mu} .
\end{aligned}
$$

Then $T(0)$ can be easily obtained from the thermal state conditions at $t=0$ as

$$
T(0)=\exp \left[-\frac{1}{2} \delta \alpha(0) \ln f(0) \bar{a}(0) \tau_{3} a(0)\right],
$$

with $\delta \alpha(t)=\alpha^{\prime}(t)-\alpha(t)$.

Since

$A_{\alpha}^{(r)}(t)^{\mu v}$

$$
\begin{aligned}
& \equiv\left\langle 0_{\alpha}\left|a_{\alpha}(t)^{\mu} \bar{a}_{\alpha}(t)^{\nu}\right| 0_{\alpha}\right\rangle \\
& =\left[\begin{array}{ll}
1+\sigma n(t) & -f^{\alpha-1}(t) n(t) \\
\sigma f^{1-\alpha}(t)[1+\sigma n(t)] & -\sigma n(t)
\end{array}\right],
\end{aligned}
$$

we can relate $A_{\alpha^{\prime}}^{(r)}(t)$ to $A_{a}^{(r)}(t)$ as follows:

$$
A_{\alpha^{\prime}}^{(r)}(t)=W(t) A_{\alpha}^{(r)}(t) W^{-1}(t)
$$

Here

$$
W(t)=\exp \left[\frac{1}{2} \tau_{3} \delta \alpha(t) \ln f(t)\right] .
$$

This shows that

$$
\begin{aligned}
& a_{\alpha^{\prime}}(t)^{\mu}=T^{-1}(0) W(t)^{\mu v} a_{\alpha}(t)^{\nu} T(0), \\
& \bar{a}_{\alpha^{\prime}}(t)^{\mu}=T^{-1}(0) \bar{a}_{\alpha}(t)^{v} W^{-1}(t)^{\nu \mu} T(0),
\end{aligned}
$$

which become

$$
\begin{aligned}
& a_{\alpha^{\prime}}(t)^{\mu}=\mathscr{T}(t) a_{\alpha}(t)^{\mu} \mathscr{T}^{-1}(t), \\
& \bar{a}_{\alpha^{\prime}}(t)^{\mu}=\mathscr{T}(t) \bar{a}_{\alpha}(t)^{\mu} \mathscr{T}^{-1}(t) .
\end{aligned}
$$

Here

$$
\mathscr{T}(t)=T^{-1}(0) T(t)
$$

with

$$
T(t)=\exp \left[-\frac{1}{2} \delta \alpha(t) \ln f(t) \bar{a}_{\alpha}(t) \tau_{3} a_{\alpha}(t)\right] .
$$

In deriving (3.9), use was made of the relations

$$
\begin{aligned}
& T(t) a_{\alpha}(t)^{\mu} T^{-1}(t)=W(t)^{\mu v} a_{\alpha}(t)^{v}, \\
& T(t) \bar{a}_{\alpha}(t)^{\mu} T^{-1}(t)=\bar{a}_{\alpha}(t)^{v} W^{-1}(t)^{\nu \mu} .
\end{aligned}
$$

Note that

$$
\mathscr{T}(0)=1 .
$$

The $\mathscr{T}(t)$ transformation changes the Hamiltonian as

$$
\hat{H}_{\alpha^{\prime}}^{0}(t)=\mathscr{T}(t) \hat{H}_{a}^{0}(t) \mathscr{T}^{-1}(t)-i \dot{T}(t) \mathscr{T}^{-1}(t) .
$$

A calculation shows that this changes only the explicit $\alpha$ in $\widehat{H}_{\alpha}^{0}(t)$ in (2.10) as $\alpha \rightarrow \alpha^{\prime}$. Thus $\delta \omega$ and $\kappa$ are independent of $\alpha$.

An operator $Q_{\alpha}$ is said to be an observable, when and only when its vacuum expectation value is independent of $\alpha$ :

$$
\left\langle 0_{\alpha}\left|Q_{\alpha}\right| 0_{\alpha}\right\rangle=\left\langle 0_{\alpha^{\prime}}\left|Q_{\alpha^{\prime}}\right| 0_{\alpha^{\prime}}\right\rangle .
$$

For our purpose it is sufficient to consider the $Q_{\alpha}$ of the form

$$
Q_{\alpha}\left(t_{1}, \ldots, t_{m}\right)=Q_{\alpha 1}\left(t_{1}\right) Q_{\alpha 2}\left(t_{2}\right) \cdots Q_{\alpha m}\left(t_{m}\right),
$$

where each of $Q_{a i}(i=1, \ldots, m)$ stands for any one of $a_{\alpha}, a_{\alpha}^{\dagger \dagger}$, $\tilde{a}_{\alpha}$, and $\tilde{a}_{\alpha}^{\dagger+}$. This is because the most general form of operators is a linear combination of the terms of the form in (3.16).

It follows from (3.9) that
$\left\langle 0_{\alpha^{\prime}}\left|Q_{\alpha^{\prime}}\right| 0_{\alpha^{\prime}}\right\rangle=e^{x}\left\langle 0_{\alpha}\left|Q_{\alpha}\right| 0_{\alpha}\right\rangle$, 
with

$$
\begin{aligned}
& x=\frac{1}{2} \sum_{i=1}^{m} \epsilon_{i} \ln \left\{\frac{f\left(t_{i}\right)^{\delta \alpha\left(t_{i}\right)}}{f(0)^{\delta \alpha(0)}}\right\}, \\
& \epsilon_{i}= \begin{cases}1, & \text { for } a_{\alpha} \text { and } \tilde{a}_{\alpha}, \\
-1, & \text { for } a_{\alpha}^{\dagger \dagger} \text { and } \tilde{a}_{\alpha}^{\dagger \dagger} .\end{cases}
\end{aligned}
$$

Comparing (3.17) and (3.15), we see that

$$
x=0
$$

is necessary and sufficient for $Q_{\alpha}$ to be an observable. In using (3.20), it is very important that $\alpha$ can vary in time.

As is seen from (3.18) and (3.20), any observable $Q_{\alpha}$ is a linear sum of products of the following operators:

$N_{\alpha}(t)=a_{\alpha}^{\dagger \dagger}(t) a_{\alpha}(t), \quad N_{\alpha}(t)=\tilde{a}_{\alpha}^{\dagger \dagger}(t) \tilde{a}_{\alpha}(t)$,

$M_{\alpha}(t)=a_{\alpha}^{\dagger \dagger}(t) \tilde{a}_{\alpha}(t), \quad \tilde{M}_{\alpha}(t)=\sigma \tilde{a}_{\alpha}^{\dagger \dagger}(t) a_{\alpha}(t)$.

Note that the operators such as $a_{\alpha}^{\dagger \dagger}\left(t_{1}\right) a_{\alpha}\left(t_{2}\right)$ with $t_{1} \neq t_{2}$ do not satisfy condition (3.20) and, therefore, are not observables.

We can summarize the above results by the statement that the observables are represented by the operators which are invariant under the following time-local dilatation:

$$
\begin{aligned}
& a_{\alpha}(t) \rightarrow e^{\theta(t)} a_{\alpha}(t), \quad \tilde{a}_{\alpha}(t) \rightarrow e^{\theta(t)} \tilde{a}_{\alpha}(t), \\
& a_{\alpha}^{\dagger \dagger}(t) \rightarrow e^{-\theta(t)} a_{\alpha}^{\dagger \dagger}(t), \quad \tilde{a}_{\alpha}^{+\dagger}(t) \rightarrow e^{-\theta(t)} \tilde{a}_{\alpha}^{+\dagger}(t) .
\end{aligned}
$$

\section{GENERAL FORMALISM FOR SEMIFREE FIELD DIVISOR}

In this section, we will consider properties of the semifree field for type II in the stationary case. This time-independent situation corresponds to the long time limit in the statistical mechanical argument. We will use the $\left[\alpha=\frac{1}{2}\right]$ representation because the general formalism for type II semifree fields is most easily constructed in this representation. Here the type II field means those fields which carry both the particle (i.e., positive-frequency wave function) and antiparticles (i.e., negative-frequency wave functions). The type I semifree field consisting of the particle only is discussed in Ref. 4.

According to (2.10) with $\alpha=\frac{1}{2}$, the Hamiltonian for the type II semifree field in a stationary situation is

$$
\begin{aligned}
\hat{H}^{0}= & \int d^{3} k\left\{\bar{a}(\mathbf{k})^{\mu}\left[\omega(\mathbf{k}) \delta^{\mu v}-i \kappa(\mathbf{k}) A(\mathbf{k})^{\mu v}\right] a(\mathbf{k})^{v}\right. \\
& \left.+\sigma^{\dagger}(\mathbf{k})^{\mu}\left[\omega(\mathbf{k}) \delta^{\mu v}-i \kappa(\mathbf{k}) A^{T}(\mathbf{k})^{\mu v}\right] b^{\dagger}(\mathbf{k})^{v}\right\},
\end{aligned}
$$

$$
A^{(a)}(\mathbf{k})^{\mu v}=\left\langle 0\left|\bar{a}(\mathbf{k})^{v} a(\mathbf{k})^{\mu}\right| 0\right\rangle=\left[\begin{array}{c}
\sigma n(\mathbf{k}) \\
\sigma \sqrt{n(\mathbf{k})[1+\sigma n(\mathbf{k})]}
\end{array}\right.
$$

and has characteristics

$$
\begin{aligned}
& {\left[A^{(r)}(\mathbf{k})\right]^{2}=A^{(r)}(\mathbf{k}),} \\
& {\left[A^{(a)}(\mathbf{k})\right]^{2}=-A^{(a)}(\mathbf{k}),} \\
& A^{(r)}(\mathbf{k}) A^{(a)}(\mathbf{k})=A^{(a)}(\mathbf{k}) A^{(r)}(\mathbf{k})=0
\end{aligned}
$$

where the matrix $A$ is defined by (2.11). We have used the thermal doublet notations both for particle $\left(a^{\mu}, \bar{a}^{\mu}\right)$ [cf. (2.1)] and antiparticle $\left(b^{\dagger \mu}, \bar{b}^{\dagger \mu}\right)$,

$$
\begin{aligned}
& b^{\dagger}(\mathbf{k})^{\mu}: b^{\dagger}(\mathbf{k})^{1}=b^{\dagger}(\mathbf{k}), \quad b^{\dagger}(\mathbf{k})^{2}=\tilde{b}(\mathbf{k}), \\
& \bar{b}^{\dagger}(\mathbf{k})^{\mu}: \bar{b}^{\dagger}(\mathbf{k})^{1}=b(\mathbf{k}), \quad \bar{b}^{\dagger}(\mathbf{k})^{2}=-\sigma \tilde{b}^{\dagger}(\mathbf{k}) .
\end{aligned}
$$

Note that the Hamiltonian and the thermal state conditions for antiparticle are obtained by the replacement $a(\mathbf{k}) \rightarrow b(\mathbf{k})$ together with $\mathbf{k} \rightarrow-\mathbf{k}$ in those for particle.

In writing (4.1), we have assumed the symmetry of the particle and antiparticle (i.e., they have the same energy spectrum $\omega$, damping parameter $\kappa$, and particle distribution $n$ ), and the isotropy of the system (i.e., the quantities $\omega, \kappa$, and $n$ are dependent on only the magnitude of $\mathbf{k}$ ). Note the property of the matrix $A$,

$$
\tau_{\sigma} A^{T} \tau_{\sigma}=A
$$

when $\alpha=\frac{1}{2}$, where $A^{T}$ indicates the transpose of $A$ and $\tau_{\sigma}$ is given in $(2.2 \mathrm{~b})$.

The Hamiltonian $\widehat{H}^{\circ}$ leads to the following equations of motion for the particle and antiparticle:

$$
\begin{aligned}
& {\left[i \partial_{t, \kappa}^{+}(\mathbf{k})^{\mu \nu}-\omega(\mathbf{k}) \delta^{\mu \nu}\right] a(t, \mathbf{k})^{\nu}=0,} \\
& \bar{a}(t, \mathbf{k})^{\mu}\left[-i \stackrel{\partial}{\partial}_{t,-\kappa}^{+}(\mathbf{k})^{\nu \mu}-\omega(\mathbf{k}) \delta^{\nu \mu}\right]=0,
\end{aligned}
$$

and

$$
\begin{aligned}
& {\left[i \partial_{t, \kappa}^{-}(\mathbf{k})^{\mu \nu}+\omega(\mathbf{k}) \delta^{\mu \nu}\right] b^{\dagger \dagger}(t, \mathbf{k})^{v}=0,} \\
& \bar{b}^{+\dagger}(t, \mathbf{k})^{\nu}\left[-i \overleftarrow{\partial}_{\bar{t},-\kappa}(\mathbf{k})^{\nu \mu}+\omega(\mathbf{k}) \delta^{\nu \mu}\right]=0,
\end{aligned}
$$

respectively, where

$$
\partial_{t, k}^{ \pm}(\mathbf{k})^{\mu v}=\partial_{t} \delta^{\mu v} \pm \kappa(\mathbf{k}) A(\mathbf{k})^{\mu v}
$$

Equations (4.5) and (4.6) are solved to give

$$
\begin{aligned}
& a(t, \mathbf{k})^{\mu}=e^{-i \omega(\mathbf{k}) t} U_{\kappa}(t, \mathbf{k})^{\mu v} a(\mathbf{k})^{v}, \\
& \bar{a}(t, \mathbf{k})^{\mu}=\bar{a}(\mathbf{k})^{v} U_{-\kappa}(t, \mathbf{k})^{v \mu} e^{i \omega(\mathbf{k}) t},
\end{aligned}
$$

and

$$
\begin{aligned}
& b^{\dagger \dagger}(t, \mathbf{k})^{\mu}=e^{i \omega(\mathbf{k}) t} U_{-\kappa}(t, \mathbf{k})^{\mu \nu} b^{\dagger}(\mathbf{k})^{\nu}, \\
& \bar{b}^{\dagger \dagger}(t, \mathbf{k})^{\mu}=\bar{b}^{\dagger}(\mathbf{k})^{\nu} U_{\kappa}(t, \mathbf{k})^{\nu \mu} e^{-i \omega(\mathbf{k}) t},
\end{aligned}
$$

respectively, where

$$
\begin{aligned}
U_{\kappa}(t, \mathbf{k}) & =\exp [-\kappa(\mathbf{k}) A(\mathbf{k}) t] \\
& =A^{(r)}(\mathbf{k}) e^{-\kappa(\mathbf{k}) t}-A^{(a)}(\mathbf{k}) e^{\kappa(\mathbf{k}) t},
\end{aligned}
$$

where the matrix $A^{(r)}$ is defined in (3.5) and $A^{(a)}$ is given by

$$
\left.\begin{array}{c}
-\sqrt{n(\mathbf{k})[1+\sigma n(\mathbf{k})]} \\
-[1+\sigma n(\mathbf{k})]
\end{array}\right]^{\mu v}
$$

$$
\begin{aligned}
& A^{(r)}(\mathbf{k})^{\mu v}-A^{(a)}(\mathbf{k})^{\mu v}=\delta^{\mu v} \\
& A^{(r)}(\mathbf{k})^{\mu v}+A^{(a)}(\mathbf{k})^{\mu v}=A(\mathbf{k})^{\mu \nu}
\end{aligned}
$$

From (4.4) and (4.5), we see that 


$$
\begin{aligned}
& -\left\{\partial_{t}^{2}+[\omega(\mathbf{k})-i \kappa(\mathbf{k}) A(\mathbf{k})]^{2}\right\}^{\mu v} a(t, \mathbf{k})^{v}=0 \\
& -\left\{\partial_{t}^{2}+[\omega(\mathbf{k})-i \kappa(\mathbf{k}) A(\mathbf{k})]^{2}\right\}^{\mu v} b^{\dagger+}(t, \mathbf{k})^{v}=0 .
\end{aligned}
$$

\section{A. The semifree field equation for a physical field}

We consider a semifree field $\varphi(x)$ of type II which satisfies the field equation

$$
\lambda_{\kappa}(\partial)^{\mu v} \varphi(x)^{v}=0,
$$

where $\partial=\left(\nabla, \partial_{t}\right)$. The field equation is called a type II equation when it can be reduced to the eigenvalue equation

$$
\left\{\partial_{t}^{2}+[\omega(-i \nabla)-i \kappa(-i \nabla) A(-i \nabla)]^{2}\right\}^{\mu v} \varphi(x)^{v}=0 .
$$

This dissipative free field equation (the equation for a semifree field) is the extension of the nondissipative free field $\left[\partial_{t}^{2}+\omega^{2}(-i \nabla)\right] \varphi=0$. Note that the form of the dissipative term is not arbitrary; it should be proportional to the matrix $A$.

\section{B. The divisor}

For the semifree field, the divisor $d_{\kappa}(\partial)$ is defined by

$$
\begin{aligned}
& \lambda_{\kappa}(\partial)^{\mu \delta} d_{\kappa}(\partial)^{\delta v} \\
& \quad=d_{\kappa}(\partial)^{\mu \delta} \lambda_{\kappa}(\partial)^{\delta v} \\
& \quad=-\left\{\partial_{t}^{2}+[\omega(-i \nabla)-i \kappa(-i \nabla) A(-i \nabla)]^{2}\right\}^{\mu v} .
\end{aligned}
$$

If we denote by $\Delta_{G}(x, y)$ any Green's functions of (4.15), $-\left\{\partial_{t}^{2}+[\omega(-i \nabla)-i \kappa(-i \nabla) A(-i \nabla)]^{2}\right\}^{\mu \delta} \Delta_{G}(x, y)^{\delta v}$

$$
=\delta^{\mu v} \delta(\mathbf{x}-\mathbf{y}) \delta(t-s),
$$

with $y=(\mathbf{y}, s)$ and $\nabla=\nabla_{x}, d_{\kappa}(\partial) \Delta_{G}(x, y)$ is a Green's function for (4.14),

$$
\lambda_{\kappa}(\partial)^{\mu \delta}\left[d_{\kappa}(\partial) \Delta_{G}(x, y)\right]^{\delta v}=\delta^{\mu v} \delta(\mathbf{x}-\mathbf{y}) \delta(t-s) .
$$

\section{The wave equation}

In the usual quantum field theory without thermal degrees of freedom, the consideration of free fields begins with finding $u(x, \mathbf{k}) a(\mathbf{k})$ for particles and $v(x, \mathbf{k}) b^{\dagger}(\mathbf{k})$ for antiparticles. Here $u(x, \mathbf{k})$ and $v(x, \mathbf{k})$ are the wave functions of positive and negative frequencies, respectively, and $x$ means $(x, t)$. These wave functions are usually determined by the free field equations. On the other hand, when the unperturbed Hamiltonian is known, they can also be obtained from the Hamiltonian. The Hamiltonian determines $a(t, \mathbf{k})$ and $b(t, \mathbf{k})$, and then the wave functions $u(x, \mathbf{k})$ and $v(x, \mathbf{k})$ are obtained through relations such as $a(t, \mathbf{k}) u(\mathbf{x}, \mathbf{k})$ $=a(\mathbf{k}) u(x, \mathbf{k})$, etc. In the case under consideration, we follow the latter method because the unperturbed Hamiltonian $\widehat{H}^{0},(4.1)$, is known.

Through the relations

$$
\begin{aligned}
& u(\mathbf{x}, \mathbf{k})^{\mu v} a(t, \mathbf{k})^{v}=u(x, \mathbf{k})^{\mu v} a(\mathbf{k})^{v}, \\
& v(\mathbf{x}, \mathbf{k})^{\mu v} b^{\dagger+}(t, \mathbf{k})^{v}=v(x, \mathbf{k})^{\mu v} b^{\dagger}(\mathbf{k})^{v},
\end{aligned}
$$

with

$$
\begin{aligned}
& u(\mathbf{x}, \mathbf{k})^{\mu v}=u(\mathbf{k})^{\mu v} e^{i \mathbf{k} \cdot \mathbf{x}}, \\
& v(\mathbf{x}, \mathbf{k})^{\mu v}=v(\mathbf{k})^{\mu \nu v} e^{-i \mathbf{k} \cdot \mathbf{x}},
\end{aligned}
$$

we have

$$
\begin{aligned}
& u(x, \mathbf{k})^{\mu \nu}=e^{-i \omega(\mathbf{k}) t+i \mathbf{k} \cdot \mathbf{x}} U_{\kappa}(t, \mathbf{k})^{\mu \delta} u(\mathbf{k})^{\delta v}, \\
& v(x, \mathbf{k})^{\mu \nu}=e^{i \omega(\mathbf{k}) t-i \mathbf{k} \cdot \mathbf{x}} U_{-\kappa}(t, \mathbf{k})^{\mu \delta} v(\mathbf{k})^{\delta v} .
\end{aligned}
$$

Here (4.7) and (4.8) have been used. We see that $u(x, \mathrm{k})$ and $v(x, \mathbf{k})$ satisfy

$$
\begin{aligned}
& {\left[i \partial_{t, \kappa}^{+}(-i \nabla)^{\mu \delta}-\omega(-i \nabla) \delta^{\mu \delta}\right] u(x, \mathbf{k})^{\delta v}=0} \\
& {\left[i \partial_{t, \kappa}^{-}(-i \nabla)^{\mu \delta}+\omega(-i \nabla) \delta^{\mu \delta}\right] v(x, \mathbf{k})^{\delta v}=0}
\end{aligned}
$$

The physical field $\varphi(x)$ is expressed as

$$
\varphi(x)^{\mu}=\int d^{3} k\left[u(x, \mathbf{k})^{\mu v} a(\mathbf{k})^{v}+v(x, \mathbf{k})^{\mu v} b^{\dagger}(\mathbf{k})^{v}\right] .
$$

Then substitution of (4.23) into the field equation (4.14) gives us wave equations

$$
\begin{aligned}
& \lambda_{\kappa}(\partial)^{\mu \delta} u(x, \mathbf{k})^{\delta v}=0, \\
& \lambda_{\kappa}(\partial)^{\mu \delta} v(x, \mathbf{k})^{\delta v}=0 .
\end{aligned}
$$

\section{The Hermitization matrix}

As we have assumed that the differential operator $\lambda_{\kappa}(\partial)$ depends on the thermal degrees of freedom (i.e., the superscripts) only through $i \kappa(-i \nabla) A(-i \nabla)$, we can easily see that it has the property

$$
\lambda_{\kappa}(-\partial) \tau_{2}^{\dagger}=\tau_{2} \lambda_{\kappa}(\partial) .
$$

In deriving (4.25), we have used the characteristic

$$
A^{T}(-i \nabla)=-\tau_{2} A(-i \nabla) \tau_{2}^{-1} .
$$

In the following, we drop the superscript unless it is needed. Equation (4.25) tells us that the matrix $\tau_{2}$ is the Hermitization matrix with respect to the thermal degrees of freedom.

The property (4.25) and the wave equations (4.24) give us

$$
\begin{aligned}
& \bar{u}(x, \mathbf{k}) \lambda_{\kappa}(-\stackrel{\leftarrow}{\partial})=0,
\end{aligned}
$$

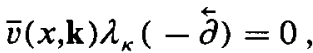

where

$$
\begin{aligned}
& \bar{u}(x, \mathbf{k})=\tau_{2} u^{\dagger}(x, \mathbf{k}) \tau_{2}^{-1}, \\
& \bar{v}(x, \mathbf{k})=\tau_{2} v^{\dagger}(x, \mathbf{k}) \tau_{2}^{-1} .
\end{aligned}
$$

Substituting (4.21) into (4.28), we have

$$
\begin{aligned}
& \bar{u}(x, \mathbf{k})^{\mu v}=\bar{u}(\mathbf{k})^{\mu \delta} U_{-\kappa}(t, \mathbf{k})^{\delta v} e^{i \omega(\mathbf{k}) t-i \mathbf{k} \cdot \mathbf{x}}, \\
& \bar{v}(x, \mathbf{k})^{\mu v}=\bar{v}(\mathbf{k})^{\mu \delta} U_{\kappa}(t, \mathbf{k})^{\delta v} e^{-i \omega(\mathbf{k}) t+i \mathbf{k} \cdot \mathbf{x}},
\end{aligned}
$$

where we have used the relation

$$
U_{\kappa}^{T}(t, \mathbf{k})=\tau_{2} U_{-\kappa}(t, \mathbf{k}) \tau_{2}^{-1},
$$

which can be obtained from (4.26). Note that the relations

$$
\begin{aligned}
& \bar{a}(t, \mathbf{k}) \bar{u}(\mathbf{x}, \mathbf{k})=\bar{a}(\mathbf{k}) \bar{u}(x, \mathbf{k}), \\
& \bar{b}^{++}(t, \mathbf{k}) \bar{v}(\mathbf{x}, \mathbf{k})=\bar{b}^{\dagger}(\mathbf{k}) \bar{v}(x, \mathbf{k}),
\end{aligned}
$$

are consistent with $(4.7 b)$ and $(4.8 b)$.

We then define 
$\bar{\varphi}(x)^{\mu}=\int d^{3} k\left[\bar{a}(\mathbf{k})^{v} \bar{u}(x, \mathbf{k})^{v \mu}+\bar{b}^{\dagger}(\mathbf{k})^{v} \bar{v}(x, \mathbf{k})^{v \mu}\right]$,

which satisfies

$$
\bar{\varphi}(x)^{v} \lambda_{\kappa}(-\overleftarrow{\partial})^{\nu \mu}=0 \text {. }
$$

The property of the divisor defined by (4.16) gives us

$$
\bar{\varphi}(x)\left\{\overleftarrow{\partial}_{t}^{2}+[\omega(i \overleftarrow{\nabla})-i \kappa(i \overleftarrow{\nabla}) A(i \overleftarrow{\nabla})]^{2}\right\}=0
$$

\section{E. The inner product of wave functions}

Let us assume

$$
\begin{aligned}
\lambda_{\kappa}(\partial)^{\mu \nu}= & \lambda^{(0)}(-i \nabla)^{\mu \gamma}+i \lambda^{(1)}(-i \nabla) \delta^{\mu \nu} \\
& \times \partial_{t}+\lambda^{(2)}(-i \nabla) \delta^{\mu \nu} \partial_{t}^{2} .
\end{aligned}
$$

The inner product of two wave functions, say $f(x)$ and $g(x)$, is defined by

$$
(f \cdot g)_{t}^{\mu v}=\int d^{3} x \bar{f}(x)^{\mu \nu} \overleftrightarrow{\Gamma}(\partial,-\partial)^{\gamma \delta} g(x)^{\delta v},
$$

where we have introduced

$$
\overleftrightarrow{\Gamma}(\partial,-\partial)^{\mu \nu}=\left[\lambda^{(1)}(-i \nabla)-i \lambda^{(2)}(-i \nabla) \overleftrightarrow{\partial}_{t}\right] \delta^{\mu \nu},
$$

with

$$
\overleftrightarrow{\partial}_{t}=\partial_{t}-\overleftarrow{\partial}_{t}
$$

When $f$ and $g$ satisfy $\lambda_{\kappa}(\partial) f(x)=\lambda_{\kappa}(\partial) g(x)=0$, we can show that $(f \cdot g)_{t}$ is independent of $t$ as follows:

$$
\begin{aligned}
\frac{d}{d t}(f \cdot g)_{t}= & \int d^{3} x \bar{f}(x)\left[\lambda^{(1)}(-i \nabla)\left(\partial_{t}+\overleftarrow{\partial}_{t}\right)\right. \\
& \left.-i \lambda^{(2)}(-i \nabla)\left(\partial_{t}+\overleftarrow{\partial}_{t}\right)\left(\partial_{t}-\overleftarrow{\partial}_{t}\right)\right] g(x) \\
= & -i \int d^{3} x \bar{f}(x)\left[\lambda_{\kappa}(\partial)-\lambda_{\kappa}(-\overleftarrow{\partial})\right] g(x) \\
= & 0,
\end{aligned}
$$

where we have performed integration by parts with respect to the space integration.

\section{F. An orthonormalized complete set of solutions of the semifree field equation (4.14)}

Being equipped with the above definition of inner product we now construct an orthonormalized complete set of solutions of the semifree field equation (4.14).

When we use $u(x, \mathbf{k})$ and $v(x, \mathbf{k})$ for $f(x)$ and $g(x)$ in (4.36), respectively, we have the following orthogonality theorem:

$$
\begin{aligned}
& \int d^{3} x \bar{u}(x, \mathbf{k}) \vec{\Gamma}_{\kappa}(\partial,-\partial) v(x, \mathbf{l})=0, \\
& \int d^{3} x \bar{v}(x, \mathbf{k}) \overleftrightarrow{\Gamma}_{\kappa}(\partial,-\partial) u(x, \mathbf{l})=0,
\end{aligned}
$$

because of the time independence of the quantities proved by (4.39).

We choose $u(x, \mathrm{k})$ and $v(x, \mathrm{k})$ to satisfy the following orthonormalization condition:

$$
\int d^{3} x \bar{u}(x, \mathbf{k})^{\mu \gamma} \vec{\Gamma}_{\kappa}(\partial,-\partial)^{\gamma \delta} u(x, \mathbf{l})^{\delta v}=\delta^{\mu v} \delta(\mathbf{k}-\mathbf{l}),
$$

$$
\int d^{3} x \bar{v}(x, \mathbf{k})^{\mu \gamma} \overleftrightarrow{\Gamma}_{\kappa}(\partial,-\partial)^{\gamma \delta} v(x, \mathbf{l})^{\delta \nu}=-\rho \delta^{\mu \nu} \delta(\mathbf{k}-\mathbf{l})
$$

In (4.41a) we chose the sign of $\lambda(\partial)$ (which determines the sign of $\overleftrightarrow{\Gamma}_{\kappa}$ ) in such a way that the left-hand side of (4.41a) is positive. Since this does not determine the sign of the quantity in (4.41b), we put the sign factor $\rho= \pm 1$ in the condition (4.41b).

Introducing

$$
\Gamma\left[k_{0}, \mathbf{k}\right]=\lambda^{(1)}(\mathbf{k})-2 k_{0} \lambda^{(2)}(\mathbf{k})=\frac{\partial}{\partial k_{0}} \lambda\left[k_{0}, \mathbf{k}\right],
$$

with

$\lambda\left[k_{0}, \mathbf{k}\right]=\lambda^{(0)}(\mathbf{k})+\lambda^{(1)}(\mathbf{k}) k_{0}-\lambda^{(2)}(\mathbf{k}) k_{0}^{2}$,

for the case given by (4.35), we see that (4.41) give

$$
\begin{aligned}
& \{\bar{u}(\mathbf{k}) \Gamma[\omega(\mathbf{k})-i \kappa(\mathbf{k}) A(\mathbf{k}), \mathbf{k}] u(\mathbf{k})\}^{\mu \nu} \\
& \quad=(2 \pi)^{-3} \delta^{\mu \nu} \\
& \{\bar{v}(\mathbf{k}) \Gamma[-\omega(\mathbf{k})+i \kappa(\mathbf{k}) A(\mathbf{k}),-\mathbf{k}] v(\mathbf{k})\}^{\mu \nu} \\
& \quad=-\rho(2 \pi)^{-3} \delta^{\mu \nu} .
\end{aligned}
$$

\section{G. The canonical sum rules}

Let us define the following two functions:

$$
\begin{aligned}
\Delta_{\kappa}^{ \pm}(x)= & \mp i \int \frac{d^{3} k}{(2 \pi)^{3}} \frac{1}{2[\omega(\mathbf{k})-i \kappa(\mathbf{k}) A(\mathbf{k})]} \\
& \times U_{ \pm \kappa}(t, \mathbf{k}) e^{\mp i[\omega(\mathbf{k}) t-\mathbf{k} \times \mathbf{x}]} .
\end{aligned}
$$

We then have

$\Delta_{\kappa}^{+}(\mathbf{x}, t)=-\Delta_{\kappa}^{-}(\mathbf{x},-t)$,

$\delta(t) \partial_{t} \Delta_{\kappa}^{ \pm}(x)=-\frac{1}{2} \delta(\mathbf{x}) \delta(t)$,

$\left\{\partial_{\imath}^{2}+[\omega(-i \nabla)-i \kappa(-i \nabla) A(-i \nabla)]^{2}\right\} \Delta^{ \pm}(x)=0$.

The last property implies that $d_{\kappa}(\partial) \Delta_{\kappa}^{ \pm}(x)$ satisfies the equation

$$
\lambda_{\kappa}(\partial)\left[d_{\kappa}(\partial) \Delta_{\kappa}^{ \pm}(x)\right]=0 .
$$

The following sum rules can be proved:

$\int d^{3} k u(x, \mathbf{k})^{\mu \delta} \bar{u}(y, \mathbf{k})^{\delta v}=i d_{\kappa}(\partial)^{\mu \delta} \Delta_{\kappa}^{+}(x-y)^{\delta v}$,

$\int d^{3} k v(x, \mathbf{k})^{v \delta} \bar{v}(y, \mathbf{k})^{\delta v}=-i \rho d_{\kappa}(\partial)^{\mu \delta} \Delta_{\kappa}^{-}(x-y)^{\delta \nu}$,

the detailed derivation of which is given in the Appendix. Since this sum rule is the basis of the equal-time canonical commutation relations, this is called the canonical sum rule. $^{8}$

Introducing

$$
\Delta_{\kappa}(x, t)=\Delta_{\kappa}^{+}(\mathbf{x}, t)+\Delta_{\kappa}^{-}(\mathbf{x}, t) \text {, }
$$


we see from (4.45) and (4.46) that

$$
\begin{aligned}
& \Delta_{\kappa}(\mathbf{x}, t) \delta(t)=0, \\
& \delta(t) \partial_{t} \Delta_{\kappa}(\mathbf{x}, t)=-\delta(\mathbf{x}) \delta(t) .
\end{aligned}
$$

Since $\Delta_{\kappa}$ satisfies the linear homogeneous differential equation of the form (4.47) which is the second order in time derivative, we have

$$
\begin{aligned}
& {\left[\left(\partial_{t}\right)^{2 n} \Delta_{\kappa}(\mathbf{x}, t)\right] \delta(t)=0,} \\
& {\left[\left(\partial_{t}\right)^{2 n+1} \Delta_{\kappa}(\mathbf{x}, t)\right] \delta(t)=0 \quad \text { for } \mathbf{x} \neq 0,}
\end{aligned}
$$

when $n$ is an integer. Thus we have

$$
\left[F(\partial) \Delta_{\kappa}(\mathbf{x}, t)\right] \delta(t)=0 \quad \text { for } \mathbf{x} \neq 0,
$$

where $F(\partial)$ stands for a sum of products of derivatives with finite powers. The combination $\left(\Delta_{\kappa}^{+}-\Delta_{\kappa}^{-}\right)$does not have this property.

\section{H. The commutation relation and statistics}

Using (4.23) and (4.32) with (4.49), we obtain

$$
\begin{aligned}
& {\left[\varphi(x)^{\mu}, \bar{\varphi}(y)^{\nu}\right]_{\sigma}} \\
& \quad=i\left\{d_{\kappa}(\partial)\left[\Delta_{\kappa}^{+}(x-y)+\sigma \rho \Delta_{\kappa}^{-}(x-y)\right]\right\}^{\mu \nu} .
\end{aligned}
$$

We now require the causality condition which states that the operators of observables should commute with each other when they refer to different points in space at a common time. Then, it follows from (4.55) and (4.56) that

$$
\sigma \rho=1 \text {. }
$$

In this way, $\rho$ determines the statistics.

\section{Projection of creation and annihilation operators}

When we are given the semifree field $\varphi(x)$ of the form (4.23), we can project out the creation and annihilation operators by means of the formulas:

$$
\begin{aligned}
& a(\mathbf{k})^{\mu}=\int d^{3} x \bar{u}(x, \mathbf{k})^{\mu \delta} \stackrel{\leftrightarrow}{\Gamma}(\partial,-\partial)^{\delta v} \varphi(x)^{v}, \\
& -\rho b^{\dagger}(\mathbf{k})^{\mu}=\int d^{3} x \bar{v}(x, \mathbf{k})^{\mu \delta} \overleftrightarrow{\Gamma}(\partial,-\partial)^{\delta v} \varphi(x)^{v}
\end{aligned}
$$

In deriving (4.58), we have used (4.40) and (4.41).

\section{J. The two-point Green's function}

The internal line in the Feynman-type diagrams is the causal two-point Green's function $\Delta_{c}(x-y)$ defined by [see (4.18)]

$$
\begin{aligned}
\Delta_{c}(x-y)_{i j}^{\mu \nu} & =d_{\kappa}(\partial)_{i j}^{\mu \delta} \Delta_{c}(x-y)^{\delta v} \\
& =-i\left\langle 0\left|T\left[\varphi(x)_{i}^{\mu} \bar{\varphi}(y)_{j}^{v}\right]\right| 0\right\rangle .
\end{aligned}
$$

Introducing the Fourier transform of $\Delta_{c}(x-y)$ with respect to the space variable by

$$
\Delta_{c}(x-y)_{i j}^{\mu v}=\int \frac{d^{3} k}{(2 \pi)^{3}} \Delta_{c}(t-s, \mathbf{k})_{i j}^{\mu v} e^{i \mathbf{k} \cdot(\mathbf{x}-\mathbf{y})},
$$

we have

$$
\begin{aligned}
\Delta_{c}(t-s, \mathbf{k})_{i j}^{\mu \nu}= & G(t-s, \mathbf{k})^{\mu \delta} \sum_{r}\left[u_{r}(\mathbf{k})_{i} \bar{u}_{r}(\mathbf{k})_{j}\right]^{\delta v} \\
& +\sigma G(s-t, \mathbf{k})^{\mu \delta} \sum_{r}\left[v_{r}(\mathbf{k})_{i} \bar{v}_{r}(\mathbf{k})_{j}\right]^{\delta v},
\end{aligned}
$$

where

$$
G(t-s, \mathbf{k})=G(t, s, \mathbf{k})
$$

with (2.23)-(2.27). Note the time independence of $B, \omega$, and $\kappa$.

Fourier transforming (4.61) further with respect to time, we have

$$
\begin{aligned}
\Delta_{c}\left(k_{0}, \mathbf{k}\right)_{i j}^{\mu \nu} & \\
= & \int_{-\infty}^{\infty} d t \Delta_{c}(t, \mathbf{k})_{i j}^{\mu v} e^{i k_{0} t} \\
= & {\left[\frac{1}{k_{0}-\omega(\mathbf{k})+i \kappa(\mathbf{k}) A(\mathbf{k})} \sum_{r} u_{r}(\mathbf{k})_{i} \bar{u}_{r}(\mathbf{k})_{j}\right]^{\mu \nu} } \\
& -\sigma\left[\frac{1}{k_{0}+\omega(\mathbf{k})-i \kappa(\mathbf{k}) A(\mathbf{k})} \sum_{r} v_{r}(\mathbf{k})_{i} \bar{v}_{r}(\mathbf{k})_{j}\right]^{\mu \nu} .
\end{aligned}
$$

In deriving (4.63), we have used the property

$$
A(\mathbf{k})=B^{-1}(\mathbf{k}) \tau_{3} B(\mathbf{k}) \text {. }
$$

Note that the existence of $i \kappa A$ in $\Delta_{c}$ means that the Feynman line is a dissipative wave even though no physical quantities dissipate in the stationary situation.

\section{K. Some examples}

A simple example of an equation of type II is given by $\lambda_{\kappa}(\partial)=-\partial_{t}^{2}-[\omega(-i \nabla)-i \kappa(-i \nabla) A(-i \nabla)]^{2}$.

In this case,

$$
\begin{aligned}
& d_{\kappa}(\partial)^{\mu v}=\delta^{\mu \nu}, \\
& \overleftrightarrow{\Gamma}_{\kappa}(\partial,-\partial)^{\mu v}=\delta^{\mu v_{i}} \overleftrightarrow{\partial}_{t}, \\
& \rho=\sigma=1 .
\end{aligned}
$$

Thus (4.43) leads to

$$
\begin{aligned}
u(\mathbf{k}) & =\bar{u}(\mathbf{k})=v(\mathbf{k})=\bar{v}(\mathbf{k}) \\
& =(2 \pi)^{-3 / 2}\{2[\omega(\mathbf{k})-i \kappa(\mathbf{k}) A(\mathbf{k})]\}^{-1 / 2} .
\end{aligned}
$$

From (4.49), the commutation relation becomes

$$
\left[\varphi(x)^{\mu}, \bar{\varphi}(y)^{\nu}\right]_{+1}=i \Delta_{\kappa}(x-y)^{\mu \nu},
$$

where $\Delta_{\kappa}(x-y)$ is defined by (4.50). If we use the property (4.52), (4.70) reduces to

$$
\left[\varphi(x)^{\mu}, \bar{\pi}(y)^{\nu}\right]_{+1} \delta(t-s)=i \delta(\mathbf{x}-\mathbf{y}) \delta(t-s),
$$

where

$$
\bar{\pi}(x)=\partial_{t} \bar{\varphi}(x) .
$$

This shows that $\varphi(x)$ and $\bar{\pi}(x)$ are canonical conjugates of each other. Note that the canonical commutation relation is based on the canonical sum rule.

The propagator $\Delta_{c}\left(k_{0}, \mathbf{k}\right)$ is given by 


$$
\Delta_{c}\left(k_{0}, \mathbf{k}\right)=B^{-1}(\mathbf{k}) \frac{1}{k_{0}^{2}-\left[\omega(\mathbf{k})-i \kappa(\mathbf{k}) \tau_{3}\right]^{2}} B(\mathbf{k}) .
$$

A more complicated example is provided by the semifree field equation of physical electrons in superconductors:

$$
\begin{aligned}
\lambda_{\kappa}(\partial)_{i j}^{\mu \nu}= & i \partial_{t} \delta_{i j} \delta^{\mu \nu}-\left[\epsilon(-i \nabla) \tau_{3 i j}-\Delta \tau_{1 i j}\right] \\
& \times \frac{\omega(-i \nabla) \delta^{\mu \nu}-i \kappa(-i \nabla) A(-i \nabla)^{\mu \nu}}{\omega(-i \nabla)},
\end{aligned}
$$

with

$$
\begin{aligned}
& \epsilon(-i \nabla)=(1 / 2 m)\left[(-i \nabla)^{2}-k_{\mathrm{F}}^{2}\right], \\
& \omega(-i \nabla)^{2}=\epsilon(-i \nabla)^{2}+\Delta^{2},
\end{aligned}
$$

where $k_{\mathrm{F}}$ is the Fermi momentum. It is easy to show that

$$
\begin{aligned}
d_{\kappa}(\partial)_{i j}^{\mu \nu}= & i \partial_{t} \delta_{i j} \delta^{\mu \nu}+\left[\epsilon(-i \nabla) \tau_{3 i j}-\Delta \tau_{1 i j}\right] \\
& \times \frac{\omega(-i \nabla) \delta^{\mu \nu}-i \kappa(-i \nabla) A(-i \nabla)^{\mu \nu}}{\omega(-i \nabla)} .
\end{aligned}
$$

From (4.37), we have

$$
\overleftrightarrow{\Gamma}_{\kappa}(\partial,-\partial)_{i j}^{\mu \nu}=\delta_{i j} \delta^{\mu \nu},
$$

and

$$
\rho=\sigma=-1 .
$$

Thus (4.43) leads to

$$
\begin{aligned}
& u(\mathbf{k})_{i}^{\mu v}=\frac{1}{(2 \pi)^{3 / 2}}\left(\begin{array}{c}
\cos \theta(\mathbf{k}) \\
-\sin \theta(\mathbf{k})
\end{array}\right)_{i}^{\sigma^{\mu v}}, \\
& v(\mathbf{k})_{i}^{\mu v}=\frac{1}{(2 \pi)^{3 / 2}}\left(\begin{array}{c}
\sin \theta(\mathbf{k}) \\
\cos \theta(\mathbf{k})
\end{array}\right)_{i}^{\sigma^{\mu \nu}},
\end{aligned}
$$

with

$$
\begin{aligned}
& \cos \theta(\mathbf{k})=\{[\omega(\mathbf{k})+\epsilon(\mathbf{k})] / 2 \omega(\mathbf{k})\}^{1 / 2}, \\
& \sin \theta(\mathbf{k})=\{[\omega(\mathbf{k})-\epsilon(\mathbf{k})] / 2 \omega(\mathbf{k})\}^{1 / 2} .
\end{aligned}
$$

The commutation relation is given by (4.56) with (4.44) and (4.76). Furthermore, we have

$$
\left[\varphi(x)_{i}^{\mu}, \bar{\varphi}(y)_{j}^{\nu}\right]_{-1} \delta(t-s)=\delta^{\mu \nu} \delta_{i j} \delta(\mathbf{x}-\mathbf{y}) \delta(t-s),
$$

which indicates that $\varphi(x)$ and $\bar{\varphi}(x)$ form a pair of canonically conjugate fields. The propagator is given by (4.63) with (4.80).

A dissipative Dirac field satisfies the semifree field equation

$$
\begin{aligned}
\lambda_{\kappa}(\partial)= & i \gamma^{0} \partial_{t}-(-i \gamma \cdot \nabla+m) \\
& \times \frac{\omega(-i \nabla)-i \kappa(-i \nabla) A(-i \nabla)}{\omega(-i \nabla)},
\end{aligned}
$$

with the Dirac $\gamma$ matrices

$$
\gamma^{0}=\beta, \quad \gamma=\beta \boldsymbol{\alpha} .
$$

The $\omega(-i \nabla)$ is defined by

$$
\omega(-i \nabla)^{2}=(-i \nabla)^{2}+m^{2} .
$$

It is easy to show that

$$
\begin{aligned}
d_{\kappa}(\partial)= & i \gamma^{0} \partial_{t}+(-i \gamma \cdot \nabla+m) \\
& \times \frac{\omega(-i \nabla)-i \kappa(-i \nabla) A(-i \nabla)}{\omega(-i \nabla)},
\end{aligned}
$$

so we have from (4.37)

$$
\overleftrightarrow{\Gamma}_{\kappa}(\partial,-\partial)_{i j}^{\mu \nu}=\left(\gamma^{0}\right)_{i j} \delta^{\mu v} \quad(i, j=1-4)
$$

and

$$
\rho=\sigma=-1 .
$$

From (4.43) we can construct the wave function as

$$
\begin{aligned}
& u(\mathbf{k})_{i}^{\mu \nu}=u(\mathbf{k})_{i} \delta^{\mu \nu}, \\
& v(\mathbf{k})_{i}^{\mu \nu}=v(\mathbf{k})_{i} \delta^{\mu v},
\end{aligned}
$$

where $u^{(r)}(\mathbf{k})_{i}$ and $v^{(r)}(\mathbf{k})_{i}(r=1,2)$ are four-component Dirac free spinors with the condition

$$
\begin{aligned}
& u^{(r) \dagger}(\mathbf{k}) \gamma_{0} u^{(s)}(\mathbf{k})=\delta^{r s} /(2 \pi)^{3}, \\
& v^{(r) \dagger}(\mathbf{k}) \gamma_{0} v^{(s)}(\mathbf{k})=\delta^{r s} /(2 \pi)^{3}, \\
& u^{(r) \dagger}(\mathbf{k}) \gamma_{0} v^{(s)}(\mathbf{k})=v^{(r)}(\mathbf{k})^{\dagger} \gamma_{0} u^{(s)}(\mathbf{k})=0 .
\end{aligned}
$$

Again the commutation relation is given by (4.56) with (4.44) and (4.86). It leads to

$$
\begin{aligned}
& {\left[\varphi(x)_{i}^{\mu}, \bar{\varphi}(y)_{j}^{v}\right]_{-1} \delta(t-s)} \\
& \quad=\delta^{\mu v}\left(\gamma_{0}\right)_{i j} \delta(\mathbf{x}-\mathbf{y}) \delta(t-s),
\end{aligned}
$$

which indicates $\varphi$ and $\bar{\varphi} \gamma_{0}$ are canonical conjugates of each other.

In this section we used the particular choice, $\alpha=\frac{1}{2}$, in constructing the canonical formalism of the semifree fields. The construction is tremendously simplified by the relation $\tau_{2} A{ }^{T} \tau_{2}=-A$, which holds if and only if $\alpha=\frac{1}{2}$. The construction of the semifree field with arbitrary $\alpha$ requires a more complex consicaration. This will be presented elsewhere.

\section{SELF-CONSISTENT EQUATIONS OF $\phi^{3}$ MODEL}

In this section we present an example of $\phi^{3}$ self-interacting real scalar in order to show how a dissipative effect can be created spontaneously in such an isolated system in TFD. (This system is assumed to be stationary below.)

In a recent paper ${ }^{7}$ we have already seen spontaneous creation of dissipation in a reservoir model, a simple solvable one, which is a system of a single harmonic oscillator bilinearly coupled to a reservoir consisting of an infinite number $N$ of harmonic oscillators. At the limit of $N \rightarrow \infty$ with fixed $\overline{\mathrm{g}}^{2}$,

$$
\bar{g}^{2}=N g^{2},
$$

$g$ being a coupling constant between the system and reservoir, our self-consistent renormalization scheme leads us to a solution in which $\kappa$ is nonvanishing and the number density of the system is determined to be at the temperature of the reservoir. The infiniteness of degrees of freedom in the reservoir, allowing the system to dissipate in it, is an essential ingredient for this. This analysis also tells us that the dissipation effect shows up as a result of the communication among the tilde and nontilde fields and, consequently, it can really be called thermal dissipation.

When we consider a nonlinear self-interacting case which is being dealt with in what follows, we should take 
account of the self-energy diagrams due to the self-interaction in the self-consistent equations. Although the system is no longer coupled to any reservoir we still have an infinite number of communication channels among the tilde and nontilde field operators due to transitions from one state to another by self-interaction. In this case as well as general nonlinearly interacting cases we may expect the thermal instability, i.e., that nontilde particles decay into multiparticles including tilde particles through many channels, because tilde particles have negative energies. So the thermal dissipation inevitably appears in nonlinearly interacting cases.

The formulation of the semifree field of type II in a stationary system has been given in Sec. IV with the choice of $\alpha=\frac{1}{2}$. We start from the model Lagrangian density for a real scalar field,

$$
\mathscr{L}=\frac{1}{2}\left[\dot{\varphi}_{0}^{2}-\varphi_{0} \omega_{0}^{2}(-i \nabla) \varphi_{0}\right]-\left(g_{0} / 3 !\right) \varphi_{0}^{3},
$$

where $\omega_{0}, g_{0}$, and $\varphi_{0}$ are bare quantities. Deriving $H$ from the canonical formula and constructing its tilde conjugate $\widetilde{H}$, we have our basic total Hamiltonian $\widehat{H}=H-\widetilde{H}$. The semifree (unperturbed renormalized) Hamiltonian $\widehat{H}^{0}$, which specifies the state vector space for realization of the field operator, should have the following form:

$\widehat{H}^{0}=\int d^{3} \mathbf{x} \frac{1}{2}\left[\dot{\bar{\varphi}}^{\mu} \dot{\varphi}^{\mu}+\bar{\varphi}^{\mu}\left\{(\omega-i \kappa A)^{2}\right\}^{\mu \nu} \varphi^{\nu}\right]$,

where $\varphi$ is the semifree (unperturbed renormalized) field [see (4.23)] and $\omega$ is a renormalized energy. Then the interaction Hamiltonian $\widehat{H}_{I}$ is unambiguously given by

$\widehat{H}_{I}=\widehat{H}-\widehat{H}^{0}=\widehat{H}_{\text {int }}+\widehat{H}_{c}$,

$\widehat{H}_{\text {int }}=\frac{g}{3 !} \int d \mathbf{x}\left[\left\{\varphi^{1}\right\}^{3}-\left\{\varphi^{2}\right\}^{3}\right]$,

$\widehat{H}_{c}=\frac{1}{2} \int d \mathbf{x} \bar{\varphi}^{\mu}\left[-\delta \omega^{2}+2 i \kappa \omega A+\kappa^{2}\right]^{\mu v} \varphi^{v}$,

where $g$ is a renormalized coupling constant and an energy counterterm $\delta \omega^{2}$ is defined by

$$
\omega_{0}^{2}=\omega^{2}-\delta \omega^{2} \text {. }
$$

In writing Eq. (5.4c), we used the relation

$$
A^{2}=1 \text {, }
$$

and suppressed the counterterms of wave function renormalization and the coupling constant renormalization since we are interested only in the two-point Green's functions in the one-loop approximation.

In order to apply the self-consistent renormalization scheme to this system, we now calculate the connected full propagator $\Delta_{c, \text { full }}$,

$$
\begin{aligned}
\Delta_{c, \text { full }}(x-y)^{\mu v}=- & i\langle 0| T\left[\varphi(x)^{\mu} \bar{\varphi}(y)^{v}\right. \\
& \left.\times \exp \left\{-i \int d t \hat{H}_{I}(t)\right\}\right]|0\rangle_{\text {con }}
\end{aligned}
$$

where the suffix conn means a connected part of the diagrams. From graphical considerations just as in the ordinary quantum field theory, $\Delta_{c, \text { full }}^{-1}$ can be expressed by the proper self-energy $\Sigma$ as

$$
\Delta_{c, \text { full }}^{-1}(k)^{\mu \nu}=\Delta_{c}^{-1}(k)^{\mu v}-\Sigma(k)^{\mu v},
$$

the free propagator $\Delta_{c}$ being given in (4.72) and $k=\left(k_{0}, \mathbf{k}\right)$. At the level of one-loop approximation, $\Sigma(k)$ is a sum of the contribution from a one-loop diagram $\Sigma_{l}(k)$ and that from counterterms,

$$
\begin{aligned}
\Sigma(k)^{\mu v}= & \Sigma_{l}(k)^{\mu \nu}+\left\{-\delta \omega^{2}+\kappa^{2}+2 i \kappa \omega A\right\}(\mathbf{k})^{\mu v}, \\
\Sigma_{l}(k)^{\mu v}= & \frac{g^{2}}{2} \int \frac{d^{4} q}{(2 \pi)^{4}} \\
& \times\left[\begin{array}{ll}
\Delta\left(q_{+}\right)^{11} \Delta\left(q_{-}\right)^{11} & -\Delta\left(q_{+}\right)^{12} \Delta\left(q_{-}\right)^{12} \\
\Delta\left(q_{+}\right)^{21} \Delta\left(q_{-}\right)^{21} & -\Delta\left(q_{+}\right)^{22} \Delta\left(q_{-}\right)^{22}
\end{array}\right], \\
& q_{ \pm}=q \pm k / 2 .
\end{aligned}
$$

For a real value of $k_{0}$, the integrations over $q_{0}$ can be performed in (5.7b), and $\Sigma_{l}$ has the matrix form of

$$
\Sigma_{l}(k)=\left[\begin{array}{cc}
L_{1}-i L_{2} & i L_{3} \\
-i L_{3} & L_{1}+i L_{2}
\end{array}\right],
$$

whose elements are further expressed as

$$
\begin{aligned}
L_{1}= & -\frac{g^{2}}{2} \int \frac{d^{3} \mathbf{q}}{(2 \pi)^{3}} \\
& \times\left\{\left(1+2 n_{+}\right) K_{(+)}^{\prime \prime}+\left(1+2 n_{-}\right) K_{(-)}^{\prime \prime}\right\},
\end{aligned}
$$

$$
\begin{aligned}
L_{2}= & -\frac{g^{2}}{2} \int \frac{d^{3} \mathbf{q}}{(2 \pi)^{3}} \\
& \times\left\{K_{(+)}^{\prime}+\left(1+2 n_{+}\right)\left(1+2 n_{-}\right) K_{(-)}^{\prime}\right\}, \\
L_{3}= & -\frac{g^{2}}{2} \int \frac{d^{3} \mathbf{q}}{(2 \pi)^{3}} \\
& \times\left\{4 \sqrt{n_{+}\left(1+n_{+}\right) n_{-}\left(1+n_{-}\right)} K_{(-)}^{\prime}\right\},
\end{aligned}
$$

with the notation of $n_{ \pm}=n(q \pm \mathrm{k} / 2)$. The real valued functions $K_{( \pm)}^{\prime}$ and $K_{( \pm)}^{\prime \prime}$ are defined through the following relations:

$$
K_{( \pm)}^{\prime}+i K_{( \pm)}^{\prime \prime} \equiv \frac{1}{2}\left(I_{1} \pm I_{2}\right),
$$

where

$$
\begin{aligned}
& I_{1}=\frac{-i\left(\Omega_{+}+\Omega_{-}\right)}{2 \Omega_{+} \Omega_{-}\left[k_{0}^{2}-\left(\Omega_{+}+\Omega_{-}\right)^{2}\right]}, \\
& I_{2}=\frac{i\left(\Omega_{+}-\Omega_{-}^{*}\right)}{2 \Omega_{+} \Omega_{-}^{*}\left[k_{0}^{2}-\left(\Omega_{+}-\Omega_{-}\right)^{2}\right]}, \\
& \Omega_{ \pm}=\omega_{ \pm}-i \kappa_{ \pm},
\end{aligned}
$$

with $\omega_{ \pm}=\omega(\mathbf{q} \pm \mathbf{k} / 2)$ and $\kappa_{ \pm}=\kappa(\mathbf{q} \pm \mathbf{k} / 2)$.

At this stage, we require the renormalization condition that the total self-energy should vanish on the energy shell, $k_{0}=\omega(\mathbf{k})$,

$$
\Sigma\left(k_{0}=\omega(\mathbf{k})\right)=0
$$

or

$$
-\Sigma_{l}\left(k_{0}=\omega(\mathbf{k})\right)=\left\{-\delta \omega^{2}+\kappa^{2}+2 i \kappa \omega A\right\}(\mathbf{k})^{\mu \nu} .
$$

The last equation is the $2 \times 2$ matrix self-consistent equation. In deriving (5.10), the "on shell" is defined by the real part of the pole of propagator. It is remarkable that this matrix equation brings us only three independent real equations because the two off-diagonal elements give the same equation, 


$$
4 \kappa \omega \sqrt{n(l+n)}=L_{3}\left(k_{0}=\omega, \mathbf{k}\right) .
$$

The real and imaginary part of the two diagonal elements of (5.10b) imply

$$
\delta \omega^{2}-\kappa^{2}=L_{1}\left(k_{0}=\omega, \mathbf{k}\right)
$$

and

$$
2 n \omega(1+2 n)=L_{2}\left(k_{0}=\omega, \mathbf{k}\right),
$$

respectively. In Eqs. (5.11), we used the notations $n=n(\mathbf{k}), \omega=\omega(\mathbf{k}), \kappa=\kappa(\mathbf{k})$, and $\delta \omega=\delta \omega(\mathbf{k})$.

The set of three equations (5.11) are the self-consistent equations for three unknown functions $\omega(\mathbf{k}), n(\mathbf{k})$, and $\kappa(\mathbf{k})$. Their solution is expressed in terms of $g_{0}$ and $m_{0}$, where

$$
\omega_{0}^{2}=\mathbf{k}^{2}+m_{0}^{2} .
$$

We have been unable so far to obtain analytic solutions except for $\kappa(\mathbf{k})=0$, because the forms of the functions $\omega(\mathbf{k}), \kappa(\mathbf{k})$, and $n(\mathbf{k})$ are not given but should be determined self-consistently. However, we expect that there are many solutions with $\kappa \neq 0$, each having different $\omega(\mathbf{k}), \kappa(\mathbf{k})$, and $n(\mathbf{k})$. The $n(\mathbf{k})$ thus obtained is determined by dynamics which includes the interaction with the thermal background fields (i.e., the tilde quanta).

The origin of $\kappa=0$ solution in our calculation can be understood in the following way. Suppose that we start our perturbation calculation with the free fields without dissipation and consider the self-energy at one-loop level. Then the decay of a nontilde particle into two nontilde particles is obviously forbidden. At first glance, we might feel that the decay of a nontilde particle into another nontilde particle and a tilde particle might be possible because the tilde particle we consider has a negative energy. The energy and momentum conservation laws read as

$$
\begin{aligned}
& \omega(\mathbf{P})=\omega\left(\mathbf{k}_{1}\right)-\omega\left(\mathbf{k}_{2}\right), \\
& \mathbf{P}=\mathbf{k}_{1}-\mathbf{k}_{2},
\end{aligned}
$$

with

$$
\omega(\mathbf{P})=\sqrt{\mathbf{P}^{2}+m^{2}} .
$$

However, further inspection of (5.13) shows that there is no solution of (5.13). This means that the $\kappa=0$ solution in the self-consistent equation can be interpreted as the result of the lowest-order perturbation expressed in terms of the usual free field without dissipation. When we consider higher orders, a nontilde particle is allowed to decay into many particle states with at least one tilde particle and, consequently, $\kappa=0$ is no longer a solution of the self-consistent equation.

\section{A SHORT SUMMARY}

It was shown that TFD offers a systematic and unified treatment of any thermal situations including nonequilibrium situations. Given a dynamics, i.e., a Hamiltonian, $H$ characterizing a system under study, one associates with it the total Hamiltonian $\widehat{H}$ as $\widehat{H}=H-\widetilde{H}$. Each thermal situation corresponds to one of various realizations of $\widehat{H}$ and is characterized by thermal state conditions at an initial time. With such initial thermal state conditions we proceed to make an interaction picture associated with it and follow a self-consistent renormalization program to determine the time evolution of $n$ as well as $\omega$ and $\kappa$. In other words, all the quantities $n, \omega$, and $\kappa$ are determined dynamically and selfconsistently. Note that here the dynamics include the communication between the nontilde particles and the thermal background fields (the tilde fields).

In the course of practical calculations we can exploit all the techniques developed in the usual field theory, because TFD is formulated as an operator field theory. Thus the use of TFD will be useful in describing the nonequilibrium system with an infinite number of degrees of freedom like in cases of quantum field systems.

Since TFD is equivalent to the other methods such as the density matrix formalism ${ }^{13}$ and the path-ordering method, ${ }^{12}$ we expect that a formulation of self-consistent renormalization for the dissipation coefficient may be needed also in other methods.

One particular flexibility in TFD can be found in the arbitrariness of the parameter $\alpha$ discussed in Sec. III. The specific choice of $\alpha$ sometimes simplifies a problem in a way similar to the choice of gauge in gauge theories.

We pointed out in Sec. I how the thermal instability in TFD is closely related to the lower unboundedness of $\widehat{H}$. It indicates that the dynamical map in terms of the asymptotic free fields is inadequate in TFD. Therefore, we have formulated the semifree fields in $\left[\alpha=\frac{1}{2}\right]$ representation for unperturbed ones, taking account of such a thermal instability from the beginning. There it is remarkable that the semifree fields has a canonical formalism. This enables us to follow a self-consistent renormalization, since the renormalization transformation is a kind of canonical transformation.

In this paper we give only the semifree fields of type II in the $\left[\alpha=\frac{1}{2}\right]$ representation. The semifree field formulation in any $[\alpha]$ representation is possible, which is explicitly shown, e.g., in Ref. 8. We think that practical calculations are performed most elegantly in the present formulation of the $\left[\alpha=\frac{1}{2}\right]$ representation because of its symmetric nature.

The infinite number of degrees of freedom combined with the lower unboundedness of $\hat{H}$ plays a central role in the present formulation of TFD and gives us a much richer structure of theory than the quantum field theory without thermal degrees of freedom. First, the fact that all thermal situations should be covered by a single Hamiltonian $\hat{H}$ is justified by the existence of inequivalent representations inherent to quantum theory with infinite degrees of freedom. Second, the nonvanishing $\kappa$ really appears as a result of infinite decay channels due to the negative energy of tilde particles. We call it spontaneous creation of dissipation, since its mechanism is analogous to the spontaneous breakdown of symmetry in quantum field theory. Third, although the complex eigenvalues of $\hat{H}^{0}$ seem to contradict the Hermiticity of $\hat{H}$, this controversy may be explained by the spontaneous breakdown of symmetry generated by the generator $H$ in which $H$ has no eigenstates and eigenvalues in the realization Fock space.

The theory developed in this paper still has many problems to be studied in the future. Above all, its application to the explicitly time-dependent case is of particular interest. There the physical content of the theory will manifest itself 
most clearly. After its accomplishment, the theory is applicable to the problem of the universe as well as various nonequilibrium problems in solid state physics. To this end, we need the formalisms of both canonical semifree fields and renormalization in time-dependent cases, on which we are preparing another paper.

\section{APPENDIX: DERIVATION OF (4.49)}

Since $d_{\kappa}(\partial) \Delta_{\kappa}^{ \pm}(x-y)$ are solutions of field equation (4.14) [see (4.48)], they can be expanded in terms of the orthornormalized solutions $u(x, \mathbf{k})$ and $v(x, \mathbf{k})$ as

$$
\begin{aligned}
& d_{\kappa}(\partial) \Delta_{\kappa}^{+}(x-y)=\int d^{3} k u(x, \mathbf{k}) C^{+}(y, \mathbf{k}) \\
& d_{\kappa}(\partial) \Delta_{\kappa}^{-}(x-y)=\int d^{3} k v(x, \mathbf{k}) C^{-}(y, \mathbf{k})
\end{aligned}
$$

When we consider the orthonormalization condition (4.41), the matrices $C^{ \pm}(y, \mathbf{k})$ can be determined as follows:

$$
C^{+}(y, \mathbf{k})=\int d^{3} k \bar{u}(x, \mathbf{k}) \overleftrightarrow{\Gamma}(\partial,-\partial) d(\partial) \Delta_{\kappa}^{+}(x-y),
$$

$$
C^{-}(y, \mathbf{k})=\int d^{3} k \bar{v}(x, \mathbf{k}) \overleftrightarrow{\Gamma}(\partial,-\partial) d(\partial) \Delta_{\kappa}^{-}(x-y)
$$

Substituting (4.44) into (A2a), we obtain

$$
\begin{aligned}
C^{+}(y, \mathbf{k})= & -i \frac{1}{2[\omega(\mathbf{k})-i \kappa(\mathbf{k}) A(\mathbf{k})]} \\
& \times \bar{u}(y, \mathbf{k}) \Gamma[\omega(\mathbf{k})-i \kappa(\mathbf{k}) A(\mathbf{k}), \mathbf{k}] \\
& \times d[\omega(\mathbf{k})-i \kappa(\mathbf{k}) A(\mathbf{k}), \mathbf{k}] .
\end{aligned}
$$

On the other hand, we see from (4.42a) that

$\Gamma\left[k_{0}, \mathbf{k}\right] d\left[k_{0}, \mathbf{k}\right]+\lambda\left[k_{0}, \mathbf{k}\right] \frac{\partial}{\partial k_{0}} d\left[k_{0}, \mathbf{k}\right]=2 k_{0}$,

where $d\left[k_{0}, \mathbf{k}\right]$ is defined by

$$
\begin{aligned}
\lambda\left[k_{0}, \mathbf{k}\right] d\left[k_{0}, \mathbf{k}\right] & =d\left[k_{0}, \mathbf{k}\right] \lambda\left[k_{0}, \mathbf{k}\right] \\
& =k_{0}^{2}-[\omega(\mathbf{k})-i \kappa(\mathbf{k}) A(\mathbf{k})]^{2}
\end{aligned}
$$

This leads to

$$
\begin{gathered}
\bar{u}(y, \mathbf{k}) \Gamma[\omega(\mathbf{k})-i \kappa(\mathbf{k}) A(\mathbf{k}), \mathbf{k}] d[\omega(\mathbf{k})-i \kappa(\mathbf{k}) A(\mathbf{k}), \mathbf{k}] \\
=2[\omega(\mathbf{k})-i \kappa(\mathbf{k}) A(\mathbf{k})] \bar{u}(y, \mathbf{k}),
\end{gathered}
$$

Thus (A3) gives

$$
C^{+}(y, \mathbf{k})=-i \bar{u}(\boldsymbol{y}, \mathbf{k}) .
$$

Similarly we have

$$
C^{-}(\boldsymbol{y}, \mathbf{k})=i \rho \bar{v}(y, \mathbf{k}) \text {. }
$$
(4.49).

Substituting (A6) into (A1), we obtain the sum rule

'T. Arimitsu and H. Umezawa, Prog. Theor. Phys. 74, 429 (1985); 77, 32 (1987).

${ }^{2}$ T. Arimitsu and H. Umezawa, Prog. Theor. Phys. 77, 53 (1987).

${ }^{3}$ T. Arimitsu, J. Pradko, and H. Umezawa, Physica A 135, 487 (1986); H. Umezawa and T. Arimitsu, Path Integrals from meV to $\mathrm{MeV}$, edited by $\mathrm{M}$. C. Gutzwiller, A. Inomata, J. R. Klauder, and L. Streit (World Scientific, Singapore, 1986), p. 217; Prog. Theor. Phys. Suppl. 86, 243 (1986); T. Arimitsu, Y. Sudo, and H. Umezawa, "Dynamical rearrangement of the thermal vacuum in TFD," Physica A, in press; H. Umezawa, Symmetries in Science II, edited by B. Gruber and R. Lenczewski (Plenum, New York, 1986), p. 517

${ }^{4}$ T. Arimitsu and H. Umezawa, J. Phys. Soc. Jpn. 55, 1475 (1986).

${ }^{5} \mathrm{~T}$. A rimitsu, M. Guida, and H. Umezawa, "Dissipative quantum field theory - thermo field dynamics-," Physica A, in press.

${ }^{6} \mathrm{~T}$. Arimitsu and $\mathrm{H}$. Umezawa, Advances on Phase Transitions and Disordered Phenomena, edited by G. Busiello et al. (World Scientific, Singapore, 1987), p. 483; T. Arimitsu, M. Guida, and H. Umezawa, Europhys. Lett. 3, 277 (1987); H. Umezawa and T. Arimitsu, Proceedings of the 2nd International Symposium on the Foundation of Quantum Mechanics-In the Light of New Technology, edited by M. Namiki et al. (Physical Society of Japan, Tokyo, 1987), p. 79.

${ }^{7}$ T. Arimitsu, H. Umezawa, Y. Yamanaka, and N. J. Papastamatiou, "Spontaneous creation of dissipation in thermo field dynamics and its examples," Physica $\mathbf{A}$, in press.

${ }^{8} \mathrm{H}$. Umezawa, Y. Yamanaka, I. Hardman, and T. Arimitsu, Wandering in the Fields, edited by K. Kawarabayashi and A. Ukawa (World Scientific, Singapore, 1987)

${ }^{9} \mathrm{H}$. Umezawa, H. Matsumoto, and M. Tachiki, Thermo Field Dynamics and Condensed States (North-Holland, Amsterdam, 1982); see also N. P. Landsman and Ch. G. van Weert, Phys. Rep. 145, 141 (1987).

${ }^{10}$ H. Umezawa, Prog. Theor. Phys. Suppl. 80, 26 (1984); H. Matsumoto, Progress in Quantum Field Theory, edited by H. Ezawa and S. Kamefuchi (North-Holland, Amsterdam, 1985), p. 171.

${ }^{11}$ N. Narnhofer, M. Requardt, and W. Thirring, Commun. Math. Phys. 92, 247 (1983)

${ }^{12}$ J. Schwinger, J. Math. Phys. 2, 407 (1961); L. V. Keldysh, Soviet Phys. JETP 20, 1018 (1965); R. A. Craig, J. Math. Phys. 9, 605 (1968); R. Mills, Propagators for Many-Particle Systems (Gordon and Breach, New York, 1969).

${ }^{13}$ For example, F. Haake, Springer Tracts in Modern Physics, edited by G. Höhler (Springer, Berlin, 1973), Vol. 66, p. 98; see also, T. Arimitsu, J. Phys. Soc. Jpn. 51, 1720 (1982). 